\title{
Nonlinear finite element analysis of orthotropic and prestressed membrane structures
}

\author{
J.G. Valdés, J. Miquel, E. Oñate* \\ International Center for Numerical Methods in Engineering (CIMNE), Technical University of Catalunya, Gran Capitán s/n, Campus Nord, 08034 Barcelona, Spain
}

\section{A R T I C L E I N F O}

\section{Article history:}

Received 13 May 2008

Accepted 21 November 2008

Available online 5 March 2009

\section{Keywords:}

Membrane structures

Orthotropy

Triangular finite elements

Prestressing

Principal fiber orientation

\begin{abstract}
A B S T R A C T
A new methodology for the geometrically nonlinear analysis of orthotropic membrane structures using triangular finite elements is presented. The approach is based on writing the constitutive equations in the principal fiber orientation of the material. A direct consequence of the fiber orientation strategy is the possibility to analyze initially out-of-plane prestressed membrane structures. An algorithm to model wrinkling behavior is also described. Examples of application to a number of membrane structures are presented.
\end{abstract}

(c) 2009 Elsevier B.V. All rights reserved.

\section{Introduction}

Membrane structures are used for many purposes in engineering and architecture. They are typically built with very light materials which are optimally used. These structures are characterized because they are only subjected to in-plane axial forces. Examples include textile covers and roofs, aircraft and space structures, parachutes, automobile airbags, sails, windmills, human tissues and long span structures.

A membrane is essentially a thin shell with no flexural stiffness. Consequently a membrane cannot resist any compression at all. However, membrane theory accounts for tension and compression stresses, and the need for a computational procedure that takes into account tension stresses only is needed. This effect is modeled in this work with a wrinkling algorithm. In membrane theory only the in-plane stress resultants are taken into account. The position of points on the two-dimensional surface in the Euclidean space gives the deformation state for a membrane. A numerical solution for membranes may be found using the finite element method. Finite element analysis of membrane structures for small deformations can be found in Zienkiewicz and Taylor [1], Cook et al. [2] or Oñate [3]. Theory for large deformations of thin membranes and shells have been proposed by Simo and Fox [4], Simo et al. [5], Bütcher et al. [6] or Braun et al. [7]. A general formulation for membranes based on curvilinear coordinates is given in Bonet et al. [8] and Lu et al. [9]. Taylor [10] proposed a large displacement finite element formulation of a membrane using three-noded triangular elements based on

\footnotetext{
* Corresponding author.

E-mail addresses: valdes@cimne.upc.edu (J.G. Valdés), onate@cimne.upc.edu
} (E. Oñate). rectangular Cartesian coordinates. Details of the various terms involved are given in Valdés [11]. This work has been generalized for different finite elements by Rossi [12].

Some membrane structures have a very low flexural stiffness that can support a small amount of compressive stress before buckling appears. In order to avoid compression stresses, membranes are prestressed. Levy and Spillers [13], Raible [14] and Gil [15] use a prestressed method to analyze membranes which are initially flat in the Euclidean space. An approach that includes curved prestressed membranes using a projection scheme can be found in Bletzinger and Wüchner [16].

In the present work, the analysis of initially curved prestressed membranes is performed using a fiber orientation strategy, which is an extension of the work of Valdés et al. $[17,18]$. A deep study for prestressed and orthotropic membranes can be found in Valdés [19]. The fiber orientation approach here presented allows to analyze orthotropic membranes.

\section{Formulation}

For the membrane formulation a curvilinear coordinate system based on differential geometry of surfaces is used $[9,16,20]$. Greek indices on the membrane mid-surface take on values of 1 and 2 in a plane stress state in the Euclidean space.

The position vector $\mathbf{X}$ on the surface in the reference configuration $\Omega_{0}$ is defined by two independent curvilinear coordinates $\xi^{1}$ and $\xi^{2}$, shown in Fig. 1, as

$\mathbf{X}=\mathbf{X}\left(\xi^{1}, \xi^{2}\right)$

The position vector $\mathbf{x}$ on the surface in the current configuration $\Omega$ is given by

$\mathbf{x}=\mathbf{x}\left(\xi^{1}, \xi^{2}, t\right)$ 

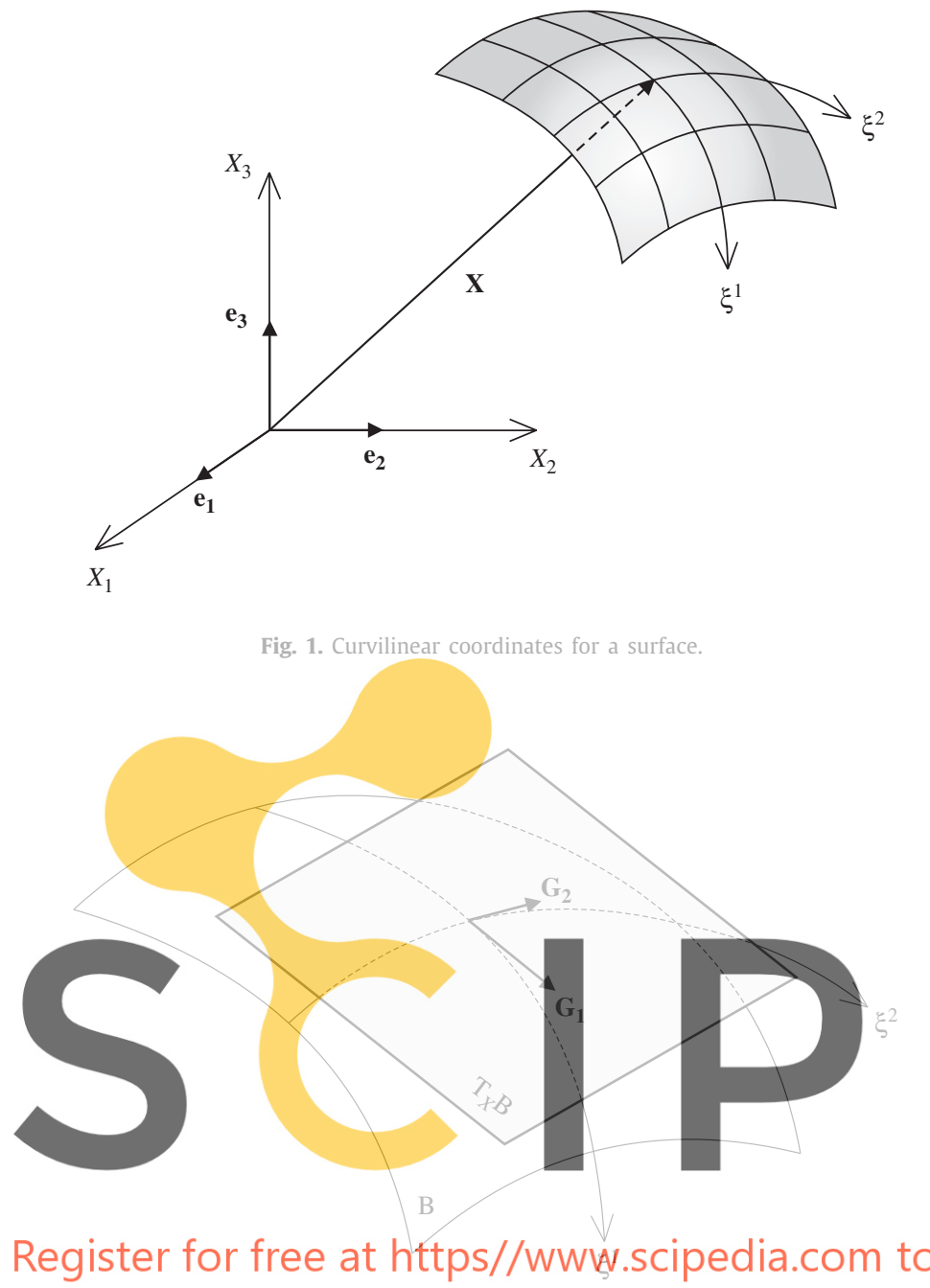

Fig. 2. Covariant base vectors forming a tangent plane.

The convected covariant base vectors of the curvilinear coordinate system on $\Omega_{0}$ and $\Omega$ are defined, respectively, as

$\mathbf{G}_{\alpha}=\frac{\partial \mathbf{X}}{\partial \xi^{\alpha}}, \quad \mathbf{g}_{\alpha}=\frac{\partial \mathbf{x}}{\partial \xi^{\alpha}}$

where the covariant base vectors $\mathbf{G}_{\alpha}$ and $\mathbf{g}_{\alpha}$ form a tangent space $\mathrm{T}_{X} \mathscr{B}$ to the membrane surface and in general they are neither unit vector nor orthogonal to each other, as shown in Fig. 2.

The surface normals are defined by

$\mathbf{G}_{3}=\mathbf{G}_{1} \times \mathbf{G}_{2}, \quad \mathbf{N}=\frac{\mathbf{G}_{3}}{\left\|\mathbf{G}_{3}\right\|}, \quad \mathbf{g}_{3}=\mathbf{g}_{1} \times \mathbf{g}_{2}, \quad \mathbf{n}=\frac{\mathbf{g}_{3}}{\left\|\mathbf{g}_{3}\right\|}$

in the reference and current configurations, respectively. The normals are normalized given a unit vector. The covariant components of the metric tensors are defined by

$G_{\alpha \beta}=\mathbf{G}_{\alpha} \cdot \mathbf{G}_{\beta}, \quad g_{\alpha \beta}=\mathbf{g}_{\alpha} \cdot \mathbf{g}_{\beta}$

for the reference and current configurations, respectively. The convected contravariant base vectors at $\Omega_{0}$ and $\Omega$ are given, respectively, by

$\mathbf{G}^{\alpha}=G^{\alpha \beta} \cdot \mathbf{G}_{\beta}, \quad \mathbf{g}^{\alpha}=g^{\alpha \beta} \cdot \mathbf{g}_{\beta}$ where the contravariant components of the metric tensors are obtained from

$\left[G^{\alpha \beta}\right]=\left[G_{\alpha \beta}\right]^{-1}, \quad\left[g^{\alpha \beta}\right]=\left[g_{\alpha \beta}\right]^{-1}$

for the corresponding configurations. When the contravariant base vectors are known, the covariant base vectors can be obtained from

$\mathbf{G}_{\alpha}=G_{\alpha \beta} \cdot \mathbf{G}^{\beta}, \quad \mathbf{g}_{\alpha}=g_{\alpha \beta} \cdot \mathbf{g}^{\beta}$

for the reference and current configurations, respectively. The covariant and contravariant base vectors define the scalar product identities

$\mathbf{G}^{\alpha} \cdot \mathbf{G}_{\beta}=\delta_{\beta}^{\alpha}, \quad \mathbf{g}^{\alpha} \cdot \mathbf{g}_{\beta}=\delta_{\beta}^{\alpha}$

where the Kronecker delta is given by

$\delta_{\beta}^{\alpha}= \begin{cases}1 & \text { when } \alpha=\beta \\ 0 & \text { otherwise }\end{cases}$

The deformation gradient tensor $\mathbf{F}$ in curvilinear coordinates is

$\mathbf{F}=\mathbf{g}_{\alpha} \otimes \mathbf{G}^{\alpha}, \quad \mathbf{F}^{T}=\mathbf{G}^{\alpha} \otimes \mathbf{g}_{\alpha}, \quad \mathbf{F}^{-1}=\mathbf{G}_{\alpha} \otimes \mathbf{g}^{\alpha}, \quad \mathbf{F}^{-T}=\mathbf{g}^{\alpha} \otimes \mathbf{G}_{\alpha}$

Substituting Eq. (11) into the Green-Lagrange strain tensor yields

$\mathbf{E}=\frac{1}{2}\left(\mathbf{F}^{T} \cdot \mathbf{F}-\mathbf{I}\right)=\frac{1}{2}\left(\mathbf{G}^{\alpha} \otimes \mathbf{g}_{\alpha} \cdot \mathbf{g}_{\beta} \otimes \mathbf{G}^{\beta}-G_{\alpha \beta} \mathbf{G}^{\alpha} \otimes \mathbf{G}^{\beta}\right)$

which components for the membrane surface in a plane stress state are

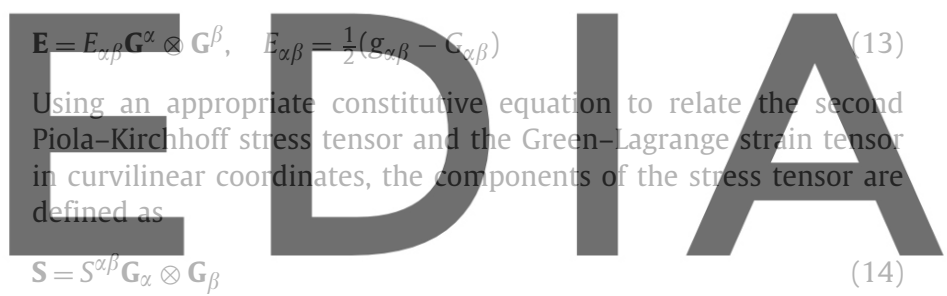

$\mathrm{S}=\mathrm{S}^{\alpha \rho} \mathrm{G}_{\alpha} \otimes \mathrm{G}$

(14)

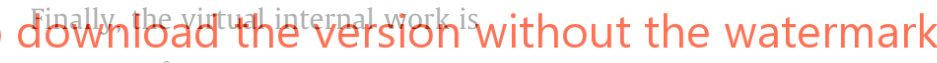 $\delta \mathbb{W}^{\text {int }}=\int_{\Omega_{0}} \delta E_{\alpha \beta} S^{\alpha \beta} \mathrm{d} \Omega_{0}$

where all the tensor components are expressed in curvilinear coordinates.

\subsection{Pressure follower forces}

An important case for geometrically nonlinear analysis of membrane structures is that of uniform normal pressure follower forces. These forces change their direction each time the normal to the surface changes in the current configuration.

Consider a membrane element with an applied uniform pressure $p$ acting on the current configuration having a pointwise normal $\mathbf{n}$. Then the traction force vector $\mathbf{t}$ is expressed as $\mathbf{p} \mathbf{n}$, and the corresponding virtual external work in the current configuration is

$\delta \mathscr{W}^{\text {ext }}=\int_{\Gamma} \delta \mathbf{u} \cdot p \mathbf{n} \mathrm{d} \Gamma$

\section{Fiber orientation}

The idea for the fiber orientation approach comes from the manufacturing process of membrane structures built with orthotropic or composite materials. A reference principal fiber direction is needed in these cases to perform the finite element analysis correctly. Even for isotropic materials the reference principal fiber direction is needed if the membrane has an initial prestressed field. With the methodology proposed here, a prestressed field for orthotropic materials is also possible. 


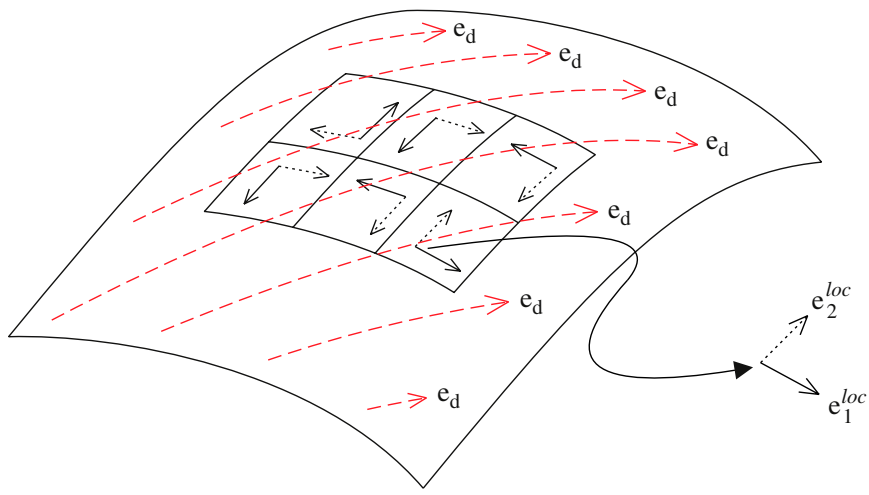

Fig. 3. Principal fiber direction and local base system.

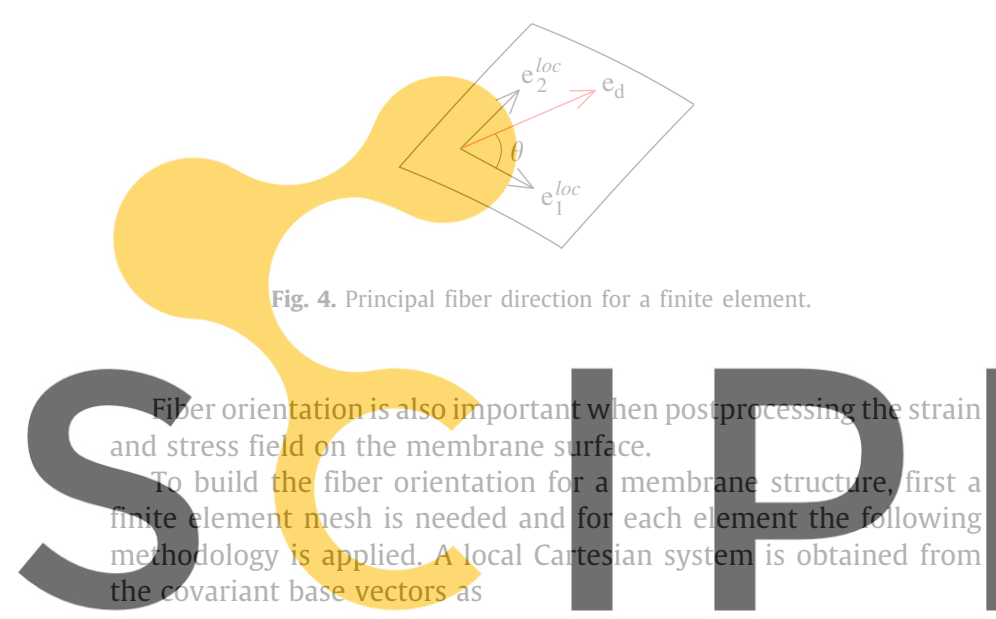

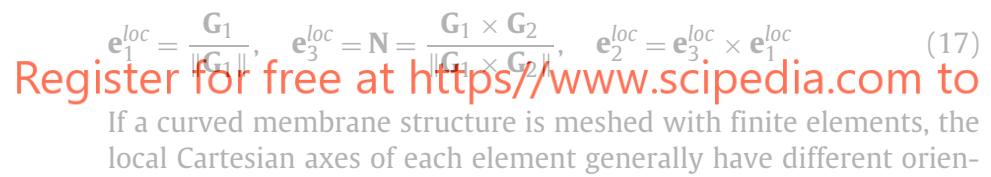
tations, even for structured meshes (Fig. 3).

If dashed-lines are the principal fiber orientations or the orthotropy direction for the material, then an angle $\theta$ is needed to rotate each local Cartesian system $\mathbf{e}_{i}^{\text {loc }}$ in order to apply the orthotropic constitutive equation of the material correctly (or the prestressed field). Suppose that $\mathbf{e}_{\mathrm{d}}$ is a given vector that defines the principal fiber orientation for a finite element, hereafter called the source finite element, and it lies on the tangent space of the element, as shown in Fig. 4. This principal fiber direction is always known at least for one finite element and it is given by the manufacturing process of the structure.

To assign correctly the principal fiber direction from a known source element to the whole mesh, first the adjacent elements of the source element must be identified, see Fig. 5(a). Next the tangent space $\mathrm{T}_{X} \mathscr{B}^{S}$ is built for the source element together with its normal called $\mathbf{N}_{S}$. For one adjacent element its tangent space $T_{X} \mathscr{B}^{N}$ is also built and its corresponding normal $\mathbf{N}_{N}$. The intersection line to both tangent spaces will be the common vector $\mathbf{X}_{S}$, see Fig. 6 .

Then a new Cartesian base system for $\mathrm{T}_{X} \mathscr{B}^{S}$ is constructed. The cross-product between the normal $\mathbf{N}_{S}$ and vector $\mathbf{X}_{S}$, yields $\mathbf{Y}_{S}$. This new vector $\mathbf{Y}_{S}$ must point towards the inside of the source element, as shown in Fig. 6.

Similarly, a new Cartesian base system for the adjacent element is built. The cross-product between the normal $\mathbf{N}_{N}$ and the vector given by the intersection line $\mathbf{X}_{N}$ yields $\mathbf{Y}_{N}$, where $\mathbf{X}_{N}=-\mathbf{X}_{S}$. This new vector $\mathbf{Y}_{N}$ must point towards the inside of the adjacent element. Note that vectors $\mathbf{X}_{S}$ and $\mathbf{Y}_{S}$ belong to the tangent space of the source element, while vectors $\mathbf{X}_{N}$ and $\mathbf{Y}_{N}$ belong to the tangent space of the adjacent element.

Once both local Cartesian base systems are defined, the next step is to choose an arbitrary point $a$ on the axis $\mathbf{X}_{S}$. Now the principal fiber direction vector $\mathbf{e}_{\mathrm{d}}$ is built over point $a$ and its intersection over the axis $\mathbf{Y}_{S}$ will be point $b$, as shown in Fig. 7.

Finally, in order to transfer the principal fiber direction to the adjacent element, the distance from the origin $o$ to point $a$ must be the same as the distance from the origin $o$ to the point $c$ located on axis $\mathbf{X}_{N}$. Also the distance from the origin $o$ to point $b$ must be the same as from the origin $o$ to point $d$ over the axis $\mathbf{Y}_{N}$. The vector from point $c$ to point $d$ will be the principal fiber direction of the neighbor element, as shown in Fig. 7.

This procedure of transferring the principal fiber direction from a source element to an adjacent element is repeated for all the elements adjacent to the source element, as shown in Fig. 5. Once each adjacent element has a principal fiber direction transferred, the procedure is repeated for the whole mesh until every element has a known principal fiber direction $\boldsymbol{e}_{\mathrm{d}}$ assigned to it. A finite element mesh with all its elements with a principal fiber direction assigned is shown in Fig. 8.

Angle $\theta$ needed to rotate each local Cartesian system for the fiber system (Fig. 4) is given from

$\sin \theta=-\mathbf{e}_{\mathrm{d}} \cdot \mathbf{e}_{2}^{l o c}, \quad \cos \theta=\mathbf{e}_{\mathrm{d}} \cdot \mathbf{e}_{1}^{l o c}$

and hence $\theta$ is found. The Cartesian system defined by the fiber orientation is named local fiber system, and it is found from the equation
$\mathbf{e}_{i}^{\text {fiber }}=\mathbf{R}(\theta, \mathbf{N}) \cdot \mathbf{e}_{i}^{\text {loc }}$
where $\mathbf{R}(\theta, \mathbf{N})$ is the Rodrigues' rotation formula that rotates a vector
an angle $\theta$ about a fixed axis specified by an unit yector, in this case
the unit hormal vector $\mathbf{N}$ to the surface element

The finite element mesh for the analysis with the fiber orientation is shown in Fig. 9. Details of the fiber orientation methodology can dodarfaload thes Mersion without the watermark

\section{Finite element discretization}

This section begins with the general discretization for membrane elements expressed in curvilinear coordinates. Next this discretization is transformed to local Cartesian coordinates which simplifies the implementation and allows the analysis of isotropic membranes. To be able to add a prestressed field or an orthotropic material behavior to the internal forces, the discretization based on the fiber orientation is presented. The implementation of the triangular threenoded membrane element is detailed in Valdés [19].

\subsection{Discretization in curvilinear coordinates}

The finite element discretization is presented for the total Lagrangian formulation. The finite element shape functions are expressed in terms of isoparametric coordinates defined in a parent element. The isoparametric coordinates are denoted by $\xi^{\alpha}$ for each element with a parent domain $\Omega_{\square}$. The shape of the parent domain depends on the type of element and the problem dimension.

The $\xi^{1}$ and $\xi^{2}$ surface coordinates of the parent element can be mapped to curvilinear coordinates for a given finite element. Therefore these coordinates are used to develop membrane elements. Consider a membrane element with parent element coordinates $\xi^{1}$ and $\xi^{2}$. Then Eq. (1) can be discretized by

$\mathbf{X}^{h}(\boldsymbol{\xi})=\sum_{I=1}^{n_{\text {node }}} N_{I}(\boldsymbol{\xi}) \mathbf{X}_{I}$ 
a

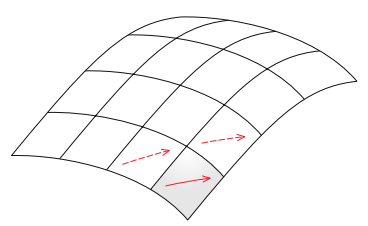

b

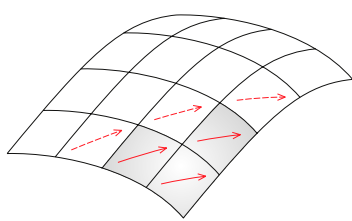

C

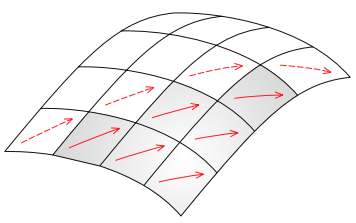

Fig. 5. Assignment of principal fiber direction to adjacent elements.

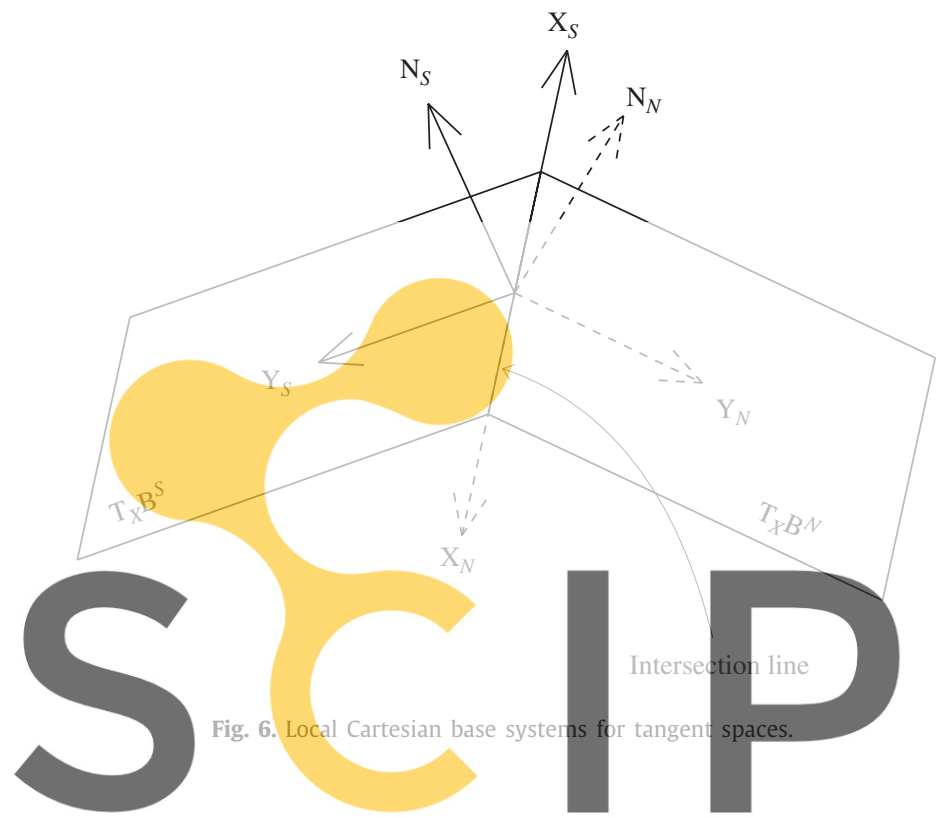

Register for free at https//www.scipedia.com to

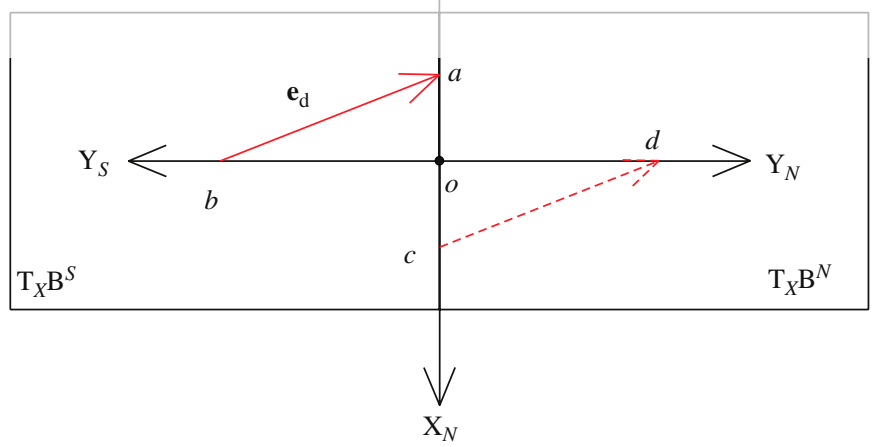

Fig. 7. Transferring reference principal fiber direction.

where $N_{I}(\xi)$ are the parent element shape functions. The position of the membrane points (Eq. (2)) and the corresponding displacements are approximated by

$\mathbf{x}^{h}(\xi, t)=\sum_{I=1}^{n_{\text {node }}} N_{I}(\xi) \mathbf{x}_{I}(t), \quad \mathbf{u}^{h}(\xi, t)=\sum_{I=1}^{n_{\text {node }}} N_{I}(\xi) \mathbf{u}_{I}(t)$

Substituting Eq. (20) into the covariant base vectors of the curvilinear coordinates in the reference configuration $\Omega_{0}$, Eq. (3), yields

$\mathbf{G}_{\alpha}=\sum_{I=1}^{n_{\text {node }}} \frac{\partial N_{I}(\boldsymbol{\xi})}{\partial \xi^{\alpha}} \mathbf{X}_{I}=\sum_{I=1}^{n_{\text {node }}} N_{I, \alpha} \mathbf{X}_{I}$

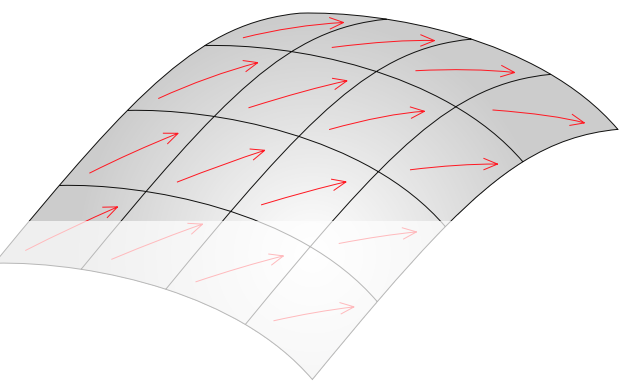

Fig. 8. Principal fiber directions in a mesh of triangular finite element.

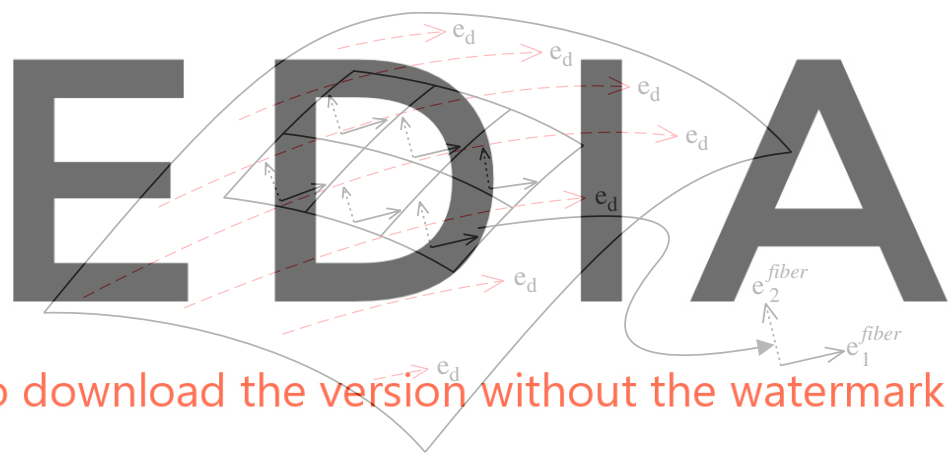

Fig. 9. Local fiber base system.

Following the same procedure, Eq. (21) is substituted into the covariant base vectors of the curvilinear coordinates in the current configuration $\Omega$, Eq. (3), to express $\mathbf{g}_{\alpha}$.

With these quantities known, the covariant components of the metric tensor $G_{\alpha \beta}$ and $g_{\alpha \beta}$ given in Eq. (5) are found. The variation of the Green-Lagrange strain tensor $\delta E_{\alpha \beta}$ becomes

$\delta E_{\alpha \beta}=\frac{1}{2} \delta g_{\alpha \beta}$

and from Eq. (5)

$\delta g_{\alpha \beta}=\delta \mathbf{g}_{\alpha} \cdot \mathbf{g}_{\beta}+\mathbf{g}_{\alpha} \cdot \delta \mathbf{g}_{\beta}$

The variation of the covariant base vectors in the current configuration results from $\mathbf{g}_{\alpha}$ yielding

$\delta \mathbf{g}_{\alpha}=\sum_{I=1}^{n_{\text {node }}} N_{I, \alpha} \delta \mathbf{u}_{I}$

where the equality $\delta \mathbf{u}_{I}=\delta \mathbf{x}_{I}$ is employed. Substituting $\mathbf{g}_{\alpha}$ and Eq. (25) into Eq. (24), and the resulting equation into Eq. (23), gives the variation of the Green-Lagrange strain tensor in terms of the virtual nodal displacement.

From the virtual internal work, Eq. (15), the internal forces for membrane elements in curvilinear coordinates for a particular 
direction $i$ and node $I$ can be expressed as

$f_{i I}^{\text {int }}=\int_{\Omega_{0}} B_{\alpha \beta i I}^{\text {cur }} S^{\alpha \beta} \mathrm{d} \Omega_{0}$

where the fourth-order strain-displacement tensor in curvilinear coordinates is given by

$B_{\alpha \beta i I}^{c u r}=\frac{1}{2}\left(N_{I, \alpha} x_{i, \beta}^{h}+N_{I, \beta} x_{i, \alpha}^{h}\right)$

where

$x_{i, \alpha}^{h}=\sum_{J=1}^{n_{\text {node }}} N_{J, \alpha} x_{i J}$

Using Voigt notation to express the internal forces in curvilinear coordinates, Eq. (26), yields

$\mathbf{f}_{I}^{\text {int }}=\int_{\Omega_{0}}\left[\mathbf{B}_{I}^{T}\right]^{\text {cur }}\{\mathbf{S}\}^{\text {cur }} \mathrm{d} \Omega_{0}$

where the membrane strain matrix $\mathbf{B}_{I}^{\text {cur }}$ is given by
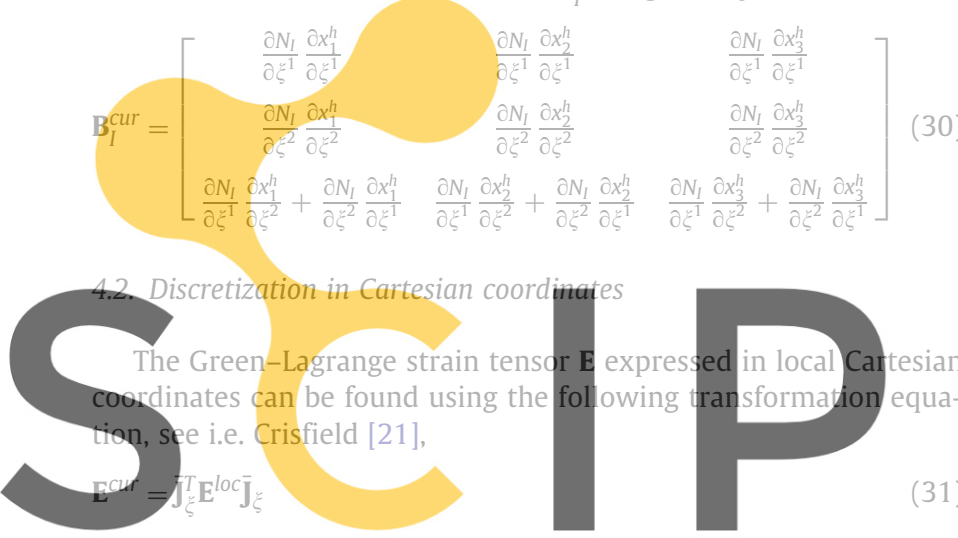

where the Jacobian transformation tensor $\bar{J}_{\xi}$ in the reference config. Registefion is defined as

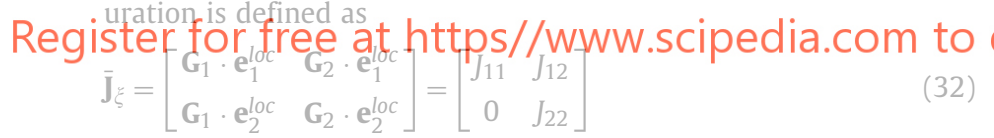

Note that the product $\mathbf{G}_{1} \cdot \mathbf{e}_{2}^{\text {loc }}=0$ because they are orthogonal vectors. From Eq. (31), the Green-Lagrange strain tensor $\mathbf{E}^{\text {loc }}$ is expressed as

$\mathbf{E}^{l o c}=\overline{\mathbf{J}}_{\xi}^{-\mathrm{T}} \mathbf{E}^{c u r} \overline{\mathbf{J}}_{\xi}^{-1}$

where the inverse of $\overline{\mathbf{J}}_{\xi}$ is given by

$\overline{\mathbf{J}}_{\xi}^{-1}=\left[\begin{array}{cc}T_{11} & T_{12} \\ 0 & T_{22}\end{array}\right]$

and

$T_{11}=\frac{1}{J_{11}}, \quad T_{12}=\frac{-J_{12}}{J_{11} J_{22}}, \quad T_{22}=\frac{1}{J_{22}}$

Eq. (33) can be written in Voigt notation yielding

$\{\mathbf{E}\}^{l o c}=[\mathbf{Q}]\{\mathbf{E}\}^{\text {cur }}$

where the transformation matrix $\mathbf{Q}$ gives the transformation from curvilinear coordinates to local Cartesian coordinates and it is defined by

$\mathbf{Q}=\left[\begin{array}{ccc}T_{11}^{2} & 0 & 0 \\ T_{12}^{2} & T_{22}^{2} & T_{22} T_{12} \\ 2 T_{11} T_{12} & 0 & T_{11} T_{22}\end{array}\right]$

From Eq. (33), the variation of the Green-Lagrange strain tensor is

$\delta \mathbf{E}^{l o c}=\overline{\mathbf{J}}_{\xi}^{-\mathrm{T}} \delta \mathbf{E}^{c u r} \overline{\mathbf{J}}_{\xi}^{-1}$ which in Voigt notation can be written as

$\{\delta \mathbf{E}\}^{l o c}=[\mathbf{Q}]\{\delta \mathbf{E}\}^{c u r}$

Consequently, the membrane strain matrix in local Cartesian coordinates and in Voigt notation is expressed as

$\mathbf{B}^{l o c}=\mathbf{Q B}^{\text {cur }}$

Finally, the internal forces local Cartesian coordinates are given in Voigt notation by

$\mathbf{f}_{I}^{\text {int }}=\int_{\Omega_{0}}\left[\mathbf{B}_{I}^{T}\right]^{c u r}\left[\mathbf{Q}^{T}\right]\{\mathbf{S}\}^{l o c} \mathrm{~d} \Omega_{0}=\int_{\Omega_{0}}\left[\mathbf{B}_{I}^{T}\right]^{l o c}\{\mathbf{S}\}^{l o c} \mathrm{~d} \Omega_{0}$

Note that this equation is defined only for isotropic materials without any prestressed field. To add the effect of a prestressed field or an orthotropic material behavior to the internal forces, the fiber orientation strategy has to be used.

\subsection{Discretization with fiber orientation}

The following relations to rotate the stress $\mathbf{S}$ and strain $\mathbf{E}$ tensors in Voigt notation are needed, see i.e. Decolon [22],

$\{\mathbf{S}\}^{l o c}=\left[\mathbf{T}_{\sigma}\right]\{\mathbf{S}\}^{\text {fib }}$

and

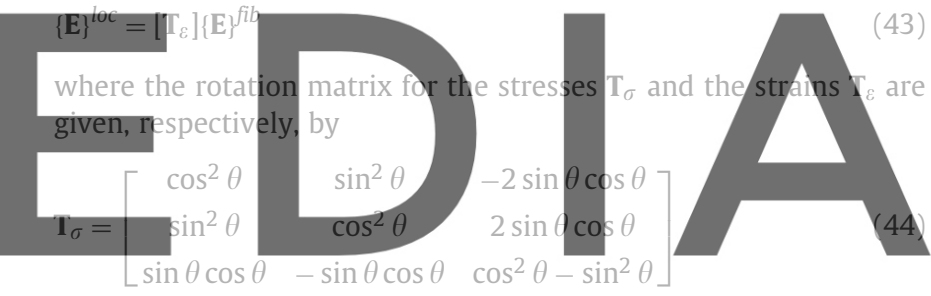

download the version without the watermark
\[ \mathbb{T}_{\varepsilon}=\left[\begin{array}{ccc}\cos ^{2} \theta & \sin ^{2} \theta & -\sin \theta \cos \theta \\ \sin ^{2} \theta & \cos ^{2} \theta & \sin \theta \cos \theta \\ 2 \sin \theta \cos \theta & -2 \sin \theta \cos \theta & \cos ^{2} \theta-\sin ^{2} \theta\end{array}\right] \]

where $\theta$ comes from the fiber orientation The inverse relationships of Eqs. (42) and (43) are given, respectively, by

$\{\mathbf{S}\}^{f i b}=\left[\mathbf{T}_{\varepsilon}^{T}\right]\{\mathbf{S}\}^{l o c}$

and

$\{\mathbf{E}\}^{f i b}=\left[\mathbf{T}_{\sigma}^{T}\right]\{\mathbf{E}\}^{l o c}$

The variation of the Green-Lagrange strain tensor expressed in the fiber orientation is

$\{\delta \mathbf{E}\}^{f i b}=\left[\mathbf{T}_{\sigma}^{T}\right]\{\delta \mathbf{E}\}^{l o c}=\left[\mathbf{T}_{\sigma}^{T}\right][\mathbf{Q}]\{\delta \mathbf{E}\}^{\text {cur }}=\left[\mathbf{T}_{\sigma}^{T}\right][\mathbf{Q}][\mathbf{B}]^{c u r}\{\delta \mathbf{u}\}$

where the membrane strain matrix expressed in the fiber orientation is given by

$\mathbf{B}^{f i b}=\mathbf{T}_{\sigma}^{T} \mathbf{Q B}^{\text {cur }}$

Finally, the internal forces expressed in the fiber orientation and in Cartesian coordinates are given by

$\mathbf{f}_{I}^{\text {int }}=\int_{\Omega_{0}}\left[\mathbf{B}_{I}^{T}\right]^{\text {cur }}\left[\mathbf{Q}^{T}\right]\left[\mathbf{T}_{\sigma}\right]\{\mathbf{S}\}^{f i b} \mathrm{~d} \Omega_{0}=\int_{\Omega_{0}}\left[\mathbf{B}_{I}^{T}\right\}^{f i b}\{\mathbf{S}\}^{f i b} \mathrm{~d} \Omega_{0}$

Note that for angle $\theta=0$, i.e. when no fiber orientation is performed, the rotation matrix $\mathbf{T}_{\sigma}=\mathbf{I}_{3 \times 3}$ and, consequently, the classical membrane element is recovered. 
Table 1

Wrinkling criterion

\begin{tabular}{lll}
\hline Stress and strain & Wrinkling & Membrane \\
\hline$S_{\mathrm{II}}>0$ & No & Taut \\
$E_{1}>0$ and $S_{\mathrm{II}} \leqslant 0$ & One axial & Wrinkled \\
$E_{\mathrm{I}} \leqslant 0$ & Two axial & Slack \\
\hline
\end{tabular}

To add a prestress field to the membrane structure once the fiber orientation process is performed, we simply add the known prestress tensor, yielding

$\mathbf{f}_{I}^{\text {int }}=\int_{\Omega_{0}}\left[\mathbf{B}_{I}^{T}\right]^{\text {fib }}\left(\{\mathbf{S}\}^{f i b}+\{\mathbf{S}\}^{\text {prestressed }}\right) \mathrm{d} \Omega_{0}$

An orthotropic analysis can also be performed once the fiber orientation process is completed, using the orthotropic plane stress constitutive equation [19].

\section{Wrinkling}

When a membrane is subjected to compression in one principal direction and to tension in the other it will buckle and many narrow wrinkles will form. Conventional membrane theory accounts for the existence of tension and compression stresses. Therefore, a difficulty in modelling membranes is to account for wrinkling phenomena that cannot be predicted by standard membrane theory. A membrane theory which accounts for wrinkling should not allow any inegative stress to appear. When a negative (co appear the membrane will wrinkle ar vanish. At any point of its surface, a memb: lowing three states. In a slack state, the ne must be in

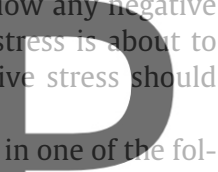
all directions orf the membrane is neither taut not slack it is a wrinkling state corresponding to uniaxial tension. In a slack or

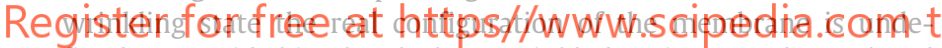
fined. To avoid this, the slack or wrinkled regions can be replaced by average smoothed pseudo-surface where material points on the real wrinkied surface are projected onto the pseudo-surface, see i.e. Lu et al. [9].

To predict the real configuration of a wrinkled membrane, a very dense finite element mesh is needed, which sometimes requires an initial perturbation, see i.e. Tessler [23]. However, from an engineering point of view, the shape of the wrinkles are not as relevant as the values of the membrane stresses.

A model capable of dealing with the wrinkling of thin membrane was introduced by Roddeman et al. [24,25]. They also introduced the correct criterion to judge the state of the membrane at a point. Table 1 shows this classification.

In this work, the wrinkling criterion based on principal stresses and principal strains introduced by Roddeman et al. [24] is used. First the membrane state is determined. If the membrane is taut, the constitutive matrix $[\mathbf{C}]$ is not modified. Then $\left[\mathbf{C}_{\mathrm{CON}}\right]=[\mathbf{C}]$ and the in-plane stresses are computed as

$\{\mathbf{S}\}^{f i b}=\left[\mathbf{C}_{\mathrm{CON}}\right]\{\mathbf{E}\}^{\text {fib }}$

If the membrane is slack, the components of the constitutive matrix $[\mathbf{C}]$ are modified yielding $\left[\mathbf{C}_{\mathrm{CON}}\right]=\mathbf{0}$ and the in-plane stress field is simply

$\{\mathbf{S}\}^{f i b}=\mathbf{0}$

Finally if the membrane wrinkles, the angle $\theta_{\sigma}$ of principal stresses is found. With this angle the stress and strain rotation matrices for stresses given by Eq. (44) and by Eq. (45) are built, where angle $\theta$ is replaced by angle $\theta_{\sigma}$. Then the constitutive matrix is rotated with the following equation:

$[\widetilde{\mathbf{C}}]=\left[\mathbf{T}_{\varepsilon}^{T}\right][\mathbf{C}]\left[\mathbf{T}_{\varepsilon}\right]$

This new rotated constitutive matrix is modified as

$\left[\mathbf{C}_{M O D}\right]=\left[\begin{array}{ccc}\widetilde{C}_{1,1} & 0 & \widetilde{C}_{1,3} \\ 0 & 0 & 0 \\ \widetilde{C}_{3,1} & 0 & \widetilde{C}_{3,3}\end{array}\right]$

Finally, this modified constitutive matrix is rotated back to its original position giving

$\left[\mathbf{C}_{\mathrm{CON}}\right]=\left[\mathbf{T}_{\sigma}\right]\left[\mathbf{C}_{\mathrm{MOD}}\right]\left[\mathbf{T}_{\sigma}^{T}\right]$

Now the in-plane stress field for the wrinkled state is computed as

$\{\mathbf{S}\}^{f i b}=\left[\mathbf{C}_{\mathrm{CON}}\right]\{\mathbf{E}\}^{\text {fib }}$

Typically the geometrically nonlinear problem is solved with a Newton-Raphson scheme with an appropriate linearization. Usually each time step $t_{i}$ is solved in 3-5 iterations. If the wrinkling algorithm presented is applied at each iteration, then the number of iterations may increase significantly.

To avoid this problem, the wrinkling algorithm is applied only for

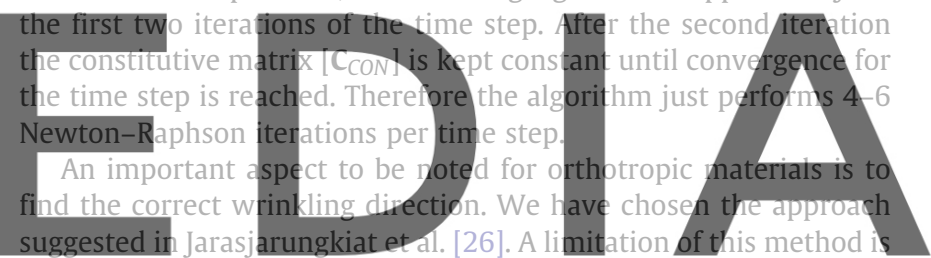

that the principal in-plane stress direction is taken as the wrinkling direction, which is only true for isotropic materials.

\section{download the version without the watermark}

6. Time integration scheme

The generalized- $\alpha$ time integration method introduced by Chung and Hulbert [27] is used. This method achieves high-frequency dissipation while minimizing unwanted low-frequency dissipation. In the generalized- $\alpha$ method, the Newmark formulas are retained, whereas the semi-discrete equations of motion are modified as follows:

$\mathbf{f}^{\text {int }}\left(\mathbf{u}_{n+\alpha_{f}^{s}}\right)+\mathbf{M} \ddot{\mathbf{u}}_{n+\alpha_{m}^{s}}=\mathbf{f}^{\text {ext }}\left(\mathbf{u}_{n+\alpha_{f}^{s}}\right)$

where

$\mathbf{u}_{n+\alpha_{f}^{s}}=\left(1-\alpha_{f}^{\mathrm{s}}\right) \mathbf{u}_{n}+\alpha_{f}^{\mathrm{s}} \mathbf{u}_{n+1}$

$\ddot{\mathbf{u}}_{n+\alpha_{m}^{s}}=\left(1-\alpha_{m}^{\mathrm{s}}\right) \ddot{\mathbf{u}}_{n}+\alpha_{m}^{\mathrm{s}} \ddot{\mathbf{u}}_{n+1}$

Low-frequency dissipation is optimal with

$\rho_{\infty}^{\mathrm{s}} \in[0,1], \quad \alpha_{f}^{\mathrm{s}}=\frac{1}{1+\rho_{\infty}^{\mathrm{s}}}, \quad \alpha_{m}^{\mathrm{s}}=\frac{2-\rho_{\infty}^{\mathrm{s}}}{1+\rho_{\infty}^{\mathrm{s}}}$

and the method is second-order accurate and posses high-frequency dissipation when

$\gamma=\frac{1}{2}+\alpha_{m}^{\mathrm{s}}-\alpha_{f}^{\mathrm{s}}, \quad \beta=\frac{1}{4}\left(1+\alpha_{m}^{\mathrm{s}}-\alpha_{f}^{\mathrm{s}}\right)^{2}$

Other possibilities for $\alpha_{f}^{s}$ and $\alpha_{m}^{s}$, as given in Chung and Hulbert [27], result in the methods of Newmark, Bossak or the $\alpha$-method. For example if $\alpha_{f}^{\mathrm{s}}=1$ and $\alpha_{m}^{\mathrm{s}}=1$, the method corresponds to the 
Newmark trapezoidal rule. The algebraic equations to be solved for $\mathbf{u}_{n+1}$ are

$$
\begin{aligned}
& \mathbf{f}^{\mathrm{int}}\left(\mathbf{u}_{n+\alpha_{f}^{\mathrm{s}}}\right)+\frac{\alpha_{m}^{\mathrm{s}}}{\beta \Delta t^{2}} \mathbf{M} \mathbf{u}_{n+1}-\mathbf{f}^{\mathrm{ext}}\left(\mathbf{u}_{n+\alpha_{f}^{\mathrm{s}}}\right) \\
& \quad=\mathbf{M}\left[\frac{\alpha_{m}^{\mathrm{s}}}{\beta \Delta t^{2}} \mathbf{u}_{n}+\frac{\alpha_{m}^{\mathrm{s}}}{\beta \Delta t} \dot{\mathbf{u}}_{n}+\left(\frac{\alpha_{m}^{\mathrm{s}}}{2 \beta}-1\right) \ddot{\mathbf{u}}_{n}\right]
\end{aligned}
$$

It is common to find external forces of the kind of pressure follower forces. Consequently, these forces are a function of the solution too.

\section{Examples}

\subsection{Prestressed membrane}

An initially prestressed square membrane is loaded by a transversal point load in its middle point. This example has been solved by

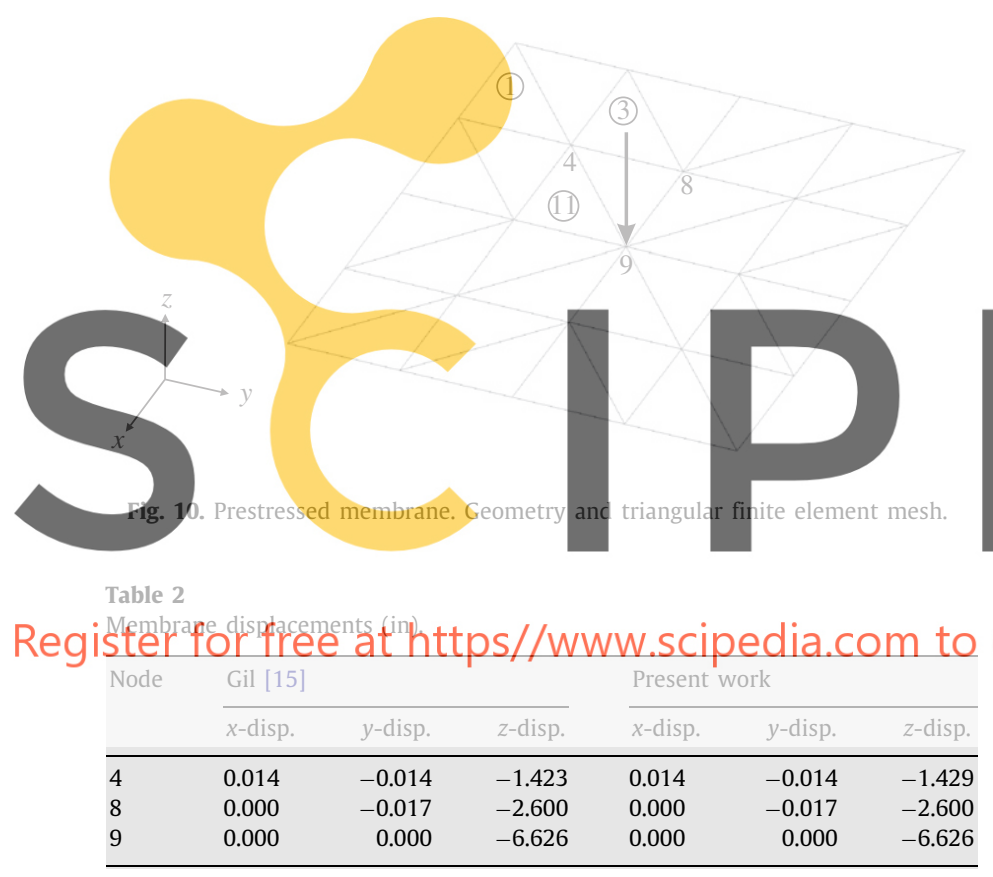

Table 3

\begin{tabular}{|c|c|c|c|c|c|c|}
\hline \multirow[t]{2}{*}{ Elements } & \multicolumn{3}{|l|}{ Gil [15] } & \multicolumn{3}{|c|}{ Present work } \\
\hline & $\sigma_{x x}$ & $\sigma_{y y}$ & $\sigma_{x y}$ & $\sigma_{x x}$ & $\sigma_{y y}$ & $\sigma_{x y}$ \\
\hline 1 & 97300 & 85164 & -2797 & 97328 & 85139 & -2794 \\
\hline 3 & 83502 & 96830 & -8631 & 83503 & 96839 & -8677 \\
\hline 11 & 144471 & 97849 & -15582 & 144812 & 97649 & -15711 \\
\hline
\end{tabular}

Membrane Cauchy stresses (psi).
Levy and Spillers [13] and Gil [15]. The point load is -10000 lb and the membrane side length is $240 \mathrm{in}$ with a thickness of $0.004167 \mathrm{in}$. Young's modulus is $E=30.0 \times 10^{6} \mathrm{psi}$ and the Poisson ratio is $v=0.3$. The prestressing field is considered to be uniform and given by $\sigma_{x x}=80000$ psi and $\sigma_{y y}=80000$ psi in the whole domain. The sides of the membrane are fixed for the analysis. Fig. 10 shows the mesh used in the analysis together with control nodes and control elements surrounded by a circle. The mesh has 32 three-noded triangular membrane elements and 25 nodes.

A comparison of the displacements at the control nodes is shown in Table 2. It can be seen that the displacements agree with the results of Gil [15] using the same mesh.

A comparison of the in-plane Cauchy stresses at the control elements is shown in Table 3, where it can be seen that the stresses are very similar too.

In this example the fiber orientation approach is performed in order to assign the same fiber orientation to the elements and also the correct prestressed values to the mesh.

For comparison purposes, the membrane is analyzed without the prestressed force which results in a vertical displacement of -9.242 in compared to -6.626 in obtained with the prestressed load. This shows the importance of prestressing in membrane structures.

\subsection{Inflation of a parachute}

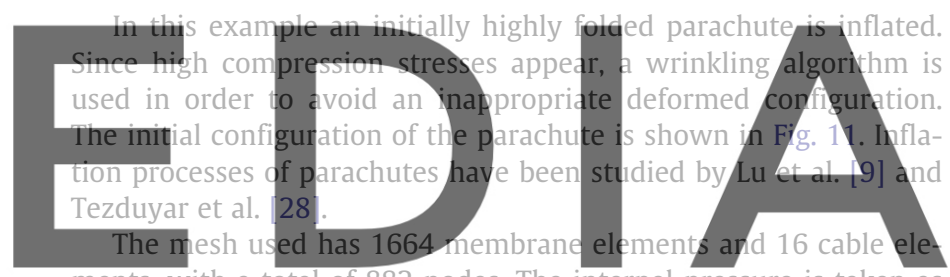
ments, with a total of 882 nodes. The internal pressure is taken as 5 Pa. Parachute material properties are Young's modulus $E=2.07 \times$

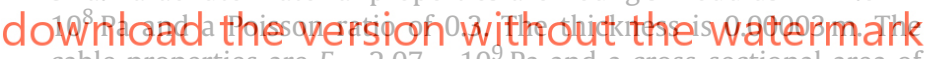
cable properties are $E=2.07 \times 10^{9} \mathrm{~Pa}$ and a cross-sectional area of $0.00013 \mathrm{~m}^{2}$.

The parachute is analyzed dynamically with the proposed vrinkling algorithm using 3000 steps and a time step size $\Delta t=0.0001 \mathrm{~s}$. A stiffness proportional damping was applied to stabilize the numerical solution.

The left picture of Fig. 12 shows the vertical displacements while the right one shows the horizontal displacements. It can be seen that the algorithm leads to a symmetric solution.

The left image of Fig. 13 shows the maximum principal stresses while the right one shows the minimum principal stresses. Both figures indicate that the wrinkling algorithm eliminates the compression stresses. Computation of the principal stresses is based on the fiber orientation approach previously described.

The same parachute is analyzed dynamically without the wrinkling algorithm. The left picture of Fig. 14 shows the
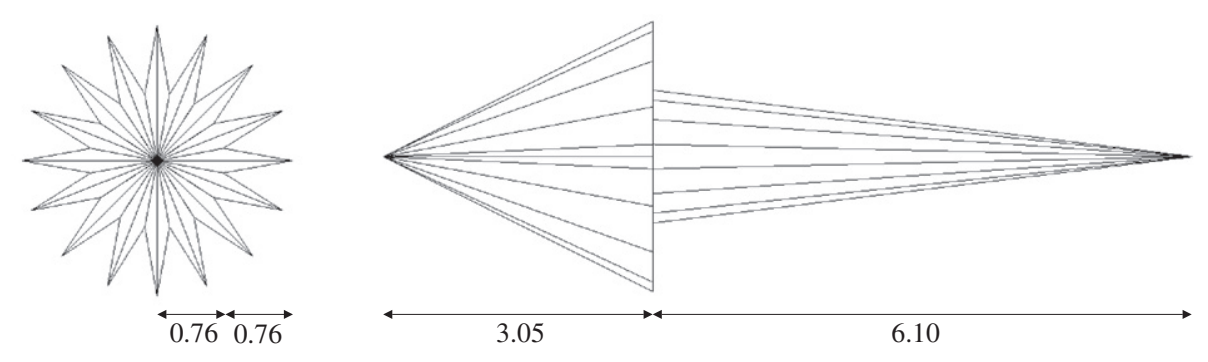

Fig. 11. Initial configuration of the parachute. Top and side views (m). 

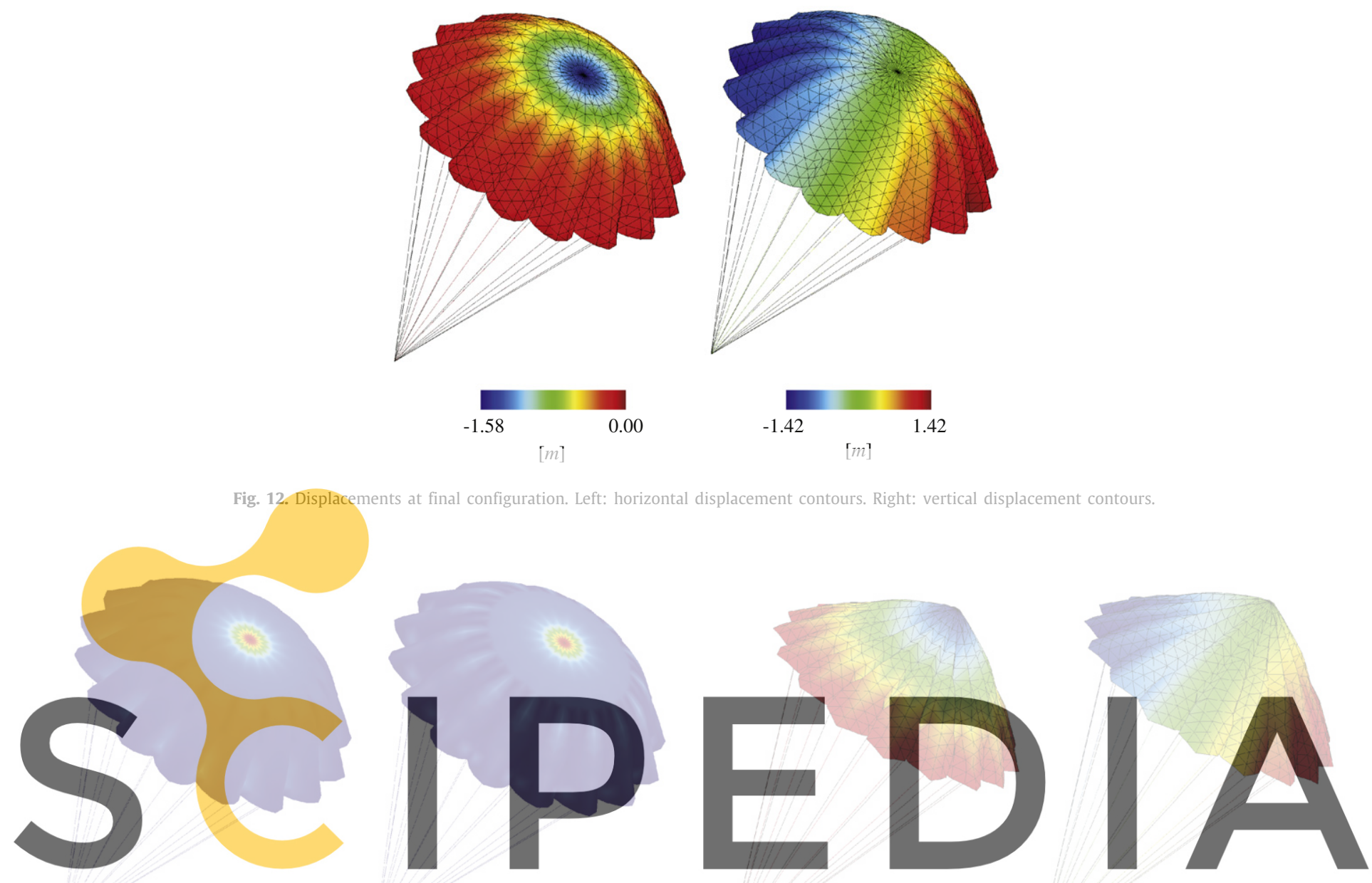

\section{Register for free at https//www.scipedia.com \\ $[\mathrm{Pa}]$ \\ $[\mathrm{Pa}]$}

Fig. 13. Principal stresses. Left: contours of maximum (tension) principal stresses. Right: contours of minimum (compression) principal stress.

vertical displacements while the right one shows the horizontal displacements.

Figs. 12 and 14 show that values for the vertical displacements are quite different while the horizontal displacements are similar. In any case the shape of both final configurations is quite different. Clearly the results obtained without the wrinkling algorithm are incorrect.

The left picture of Fig. 15 shows the maximum principal stresses while the right image of Fig. 15 shows the minimum principal stresses. In both figures it can be seen that compression stresses appear due to the use of conventional membrane theory.

Fig. 16 shows the two final configurations of the parachute. One including the wrinkling algorithm and the other without it. Note that the shape of the inflated parachute is incorrect if the wrinkling algorithm is not used. Also the maximum principal stresses are very different. In the analysis with the wrinkling algorithm there are no compression stresses, just as it really happens.

In conclusion, a standard membrane analysis excluding a wrinkling algorithm leads to an unreal final configuration due to the presence of compression stresses.
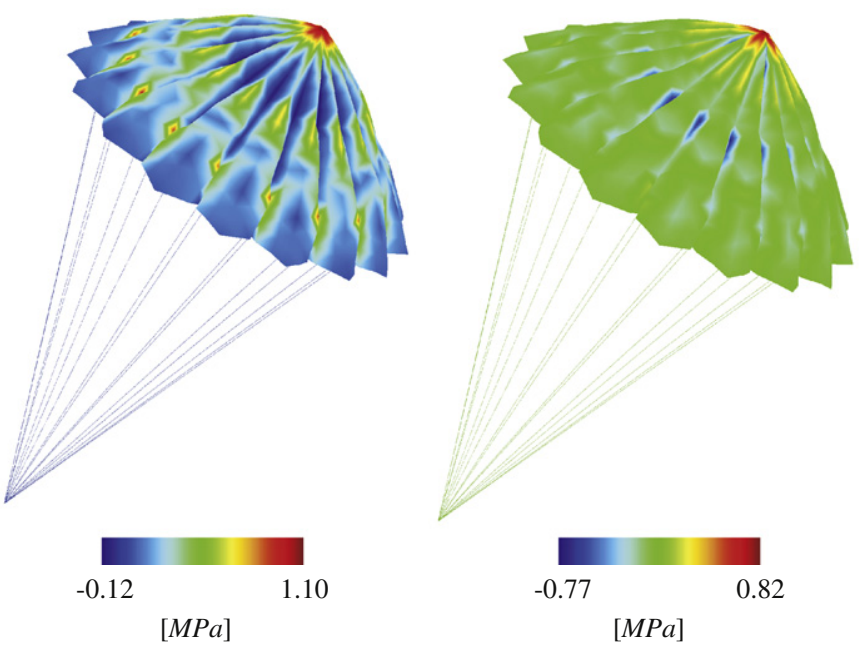

Fig. 15. Principal stresses. 


\subsection{Orthotropic spinnaker}

Usually sails are manufactured with composite materials, which sometimes are modeled with isotropic or orthotropic models for simplicity. Fig. 17 shows different materials for sails. Fig. 19 shows the manufacturing process for a spinnaker sail.

A sail is built assembling single parts of the cutting pattern which are sewed and glued together. Each one of the parts has reinforcement fibers at different directions for optimization of the sail shape. In the case of a spinnaker, their orientations are shown in Fig. 18.

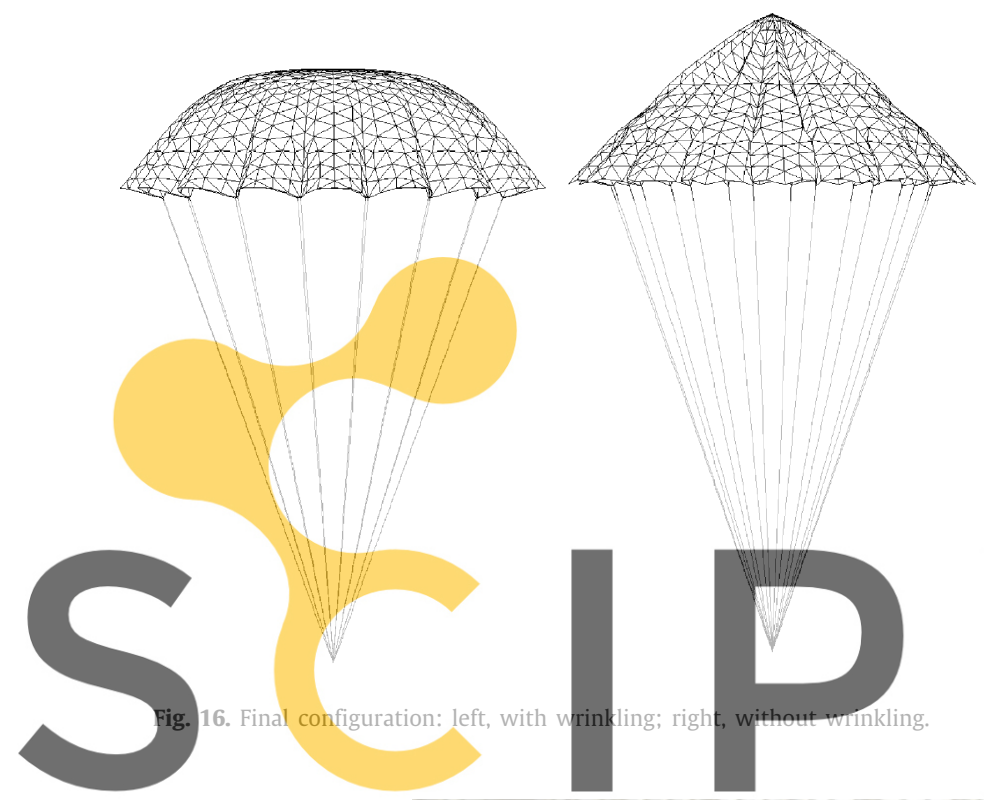

The objective of this example is to show how a sail built with an orthotropic material can be analyzed, including different local orientations for the material.

Following the methodology given in this work for assigning the fiber orientation, Fig. 19 shows vectors of the local $x$-direction for the analysis, which is the optimal fiber orientation for a spinnaker.

Orthotropic material properties are Young's modulii $E_{x}=$ $1100 \mathrm{~N} / \mathrm{mm}^{2}, E_{y}=385 \mathrm{~N} / \mathrm{mm}^{2}$, Poisson ratios: $v_{x y}=035, v_{y x}=0.1225$ and shear modulus $G_{x y}=220 \mathrm{~N} / \mathrm{mm}^{2}$. A uniform follower pressure load of $20 \mathrm{~N} / \mathrm{m}^{2}$ is applied. The larger width dimension is $6.5 \mathrm{~m}$, with a hight of $14.0 \mathrm{~m}$ and thickness of $0.1 \mathrm{~mm}$. The structure is discretized with 686 three-noded triangular membrane elements and 384 nodes.

Fig. 20 shows contour values of the second Piola-Kirchhoff stresses. On the left part of the figure, contour values for $S_{x x}$ are plotted, where the local direction for $x x$ is given by the optimal orientation of the fibers, as illustrated in Fig. 19. The maximum local values for $S_{x x}$ are $25.87 \mathrm{MPa}$ and the minimum one is $0.64 \mathrm{Mpa}$.

The right part of Fig. 20 shows values for the 1st principal component $S_{1}$ of the second Piola-Kirchhoff stress tensor with a maximum of $25.92 \mathrm{MPa}$ and a minimum of $0.68 \mathrm{MPa}$. In this case, both stress fields are almost identical because the orientation of the principal fiber of the material follows the directions of the 1 st principal component of the stress tensor, as shown in Fig. 21.

On the contrary, if we choose the horizontal direction for the fiber orientation, the values for $S_{x x}$ have a maximum of $8.22 \mathrm{MPa}$ and a minimum of $-0.57 \mathrm{MPa}$. With this horizontal fiber orientation,
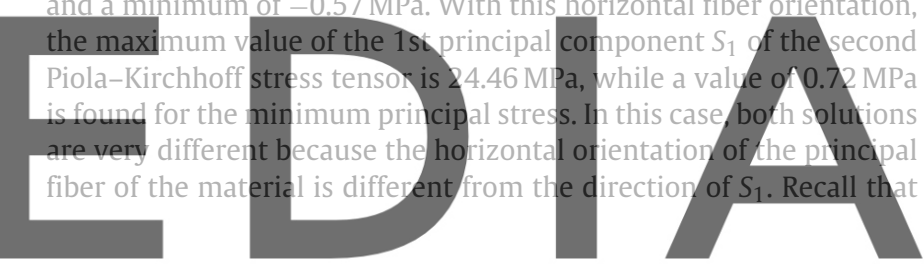

Register for free at https//www.scipedia.com to download the version without the watermark
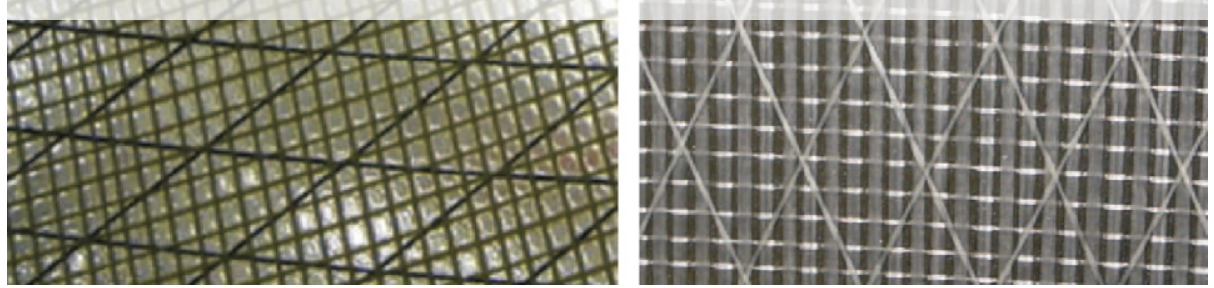

Fig. 17. Materials to manufacture sails.
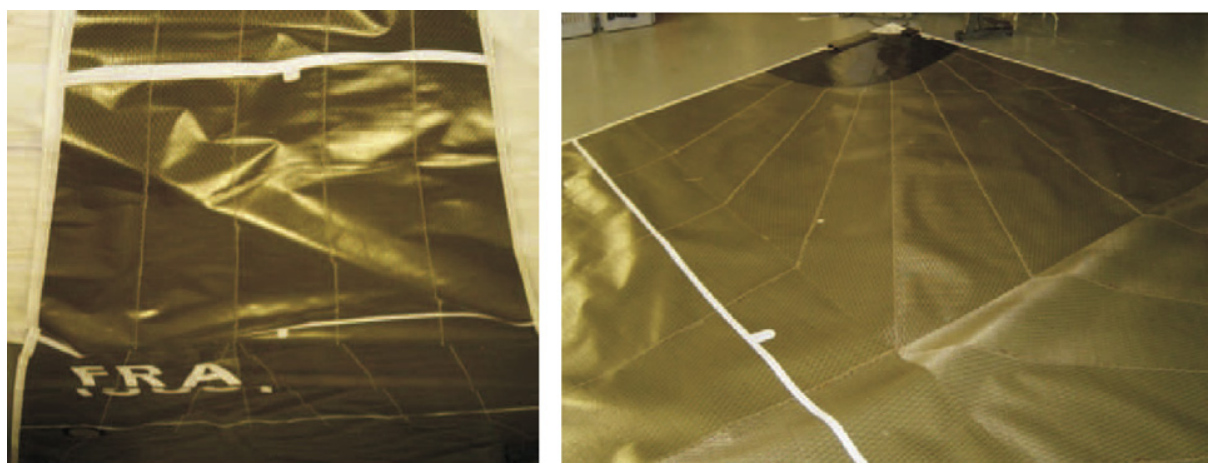

Fig. 18. Spinnaker manufacturing process. 


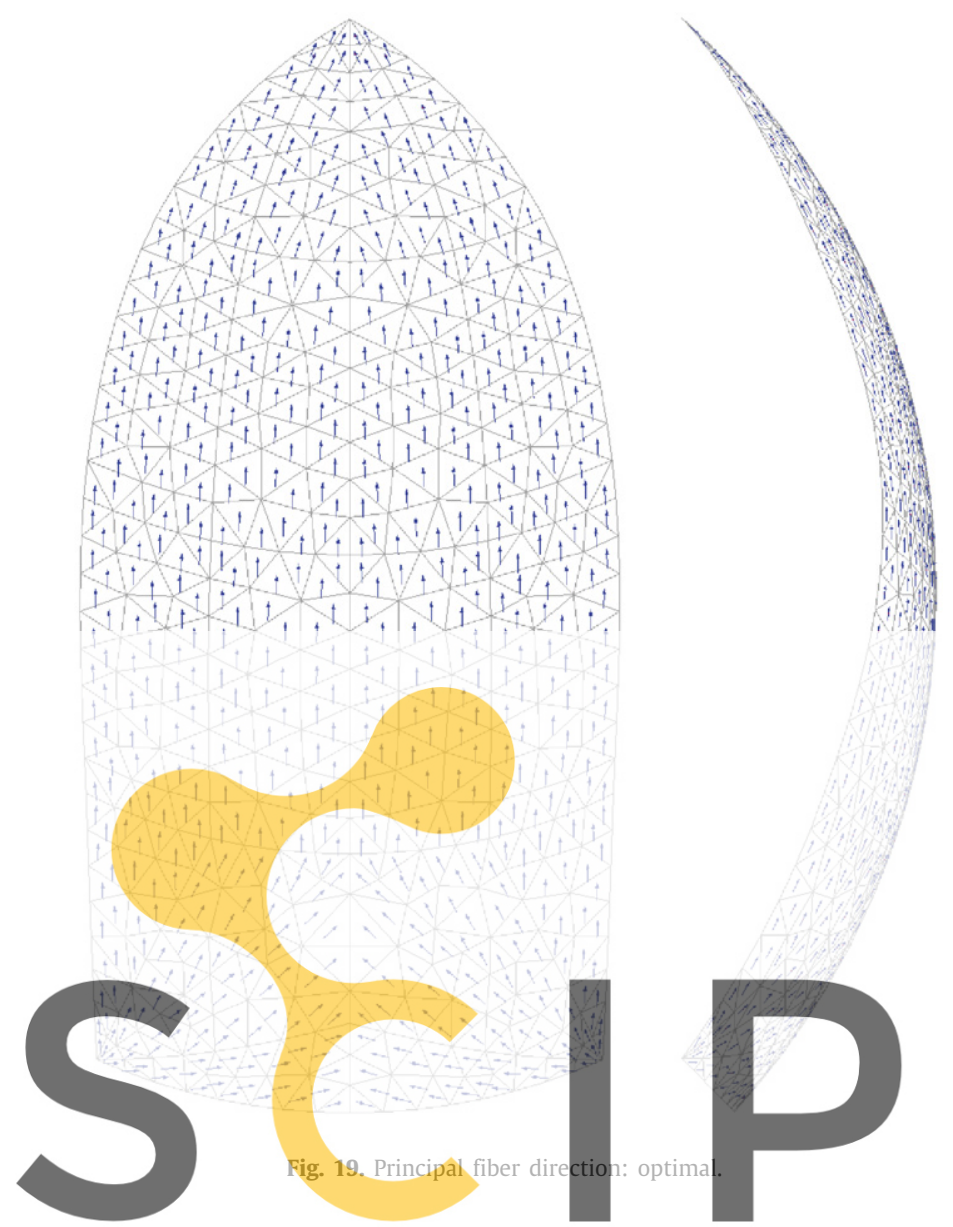

\section{Register for free at https//www.scipedia.com to d}
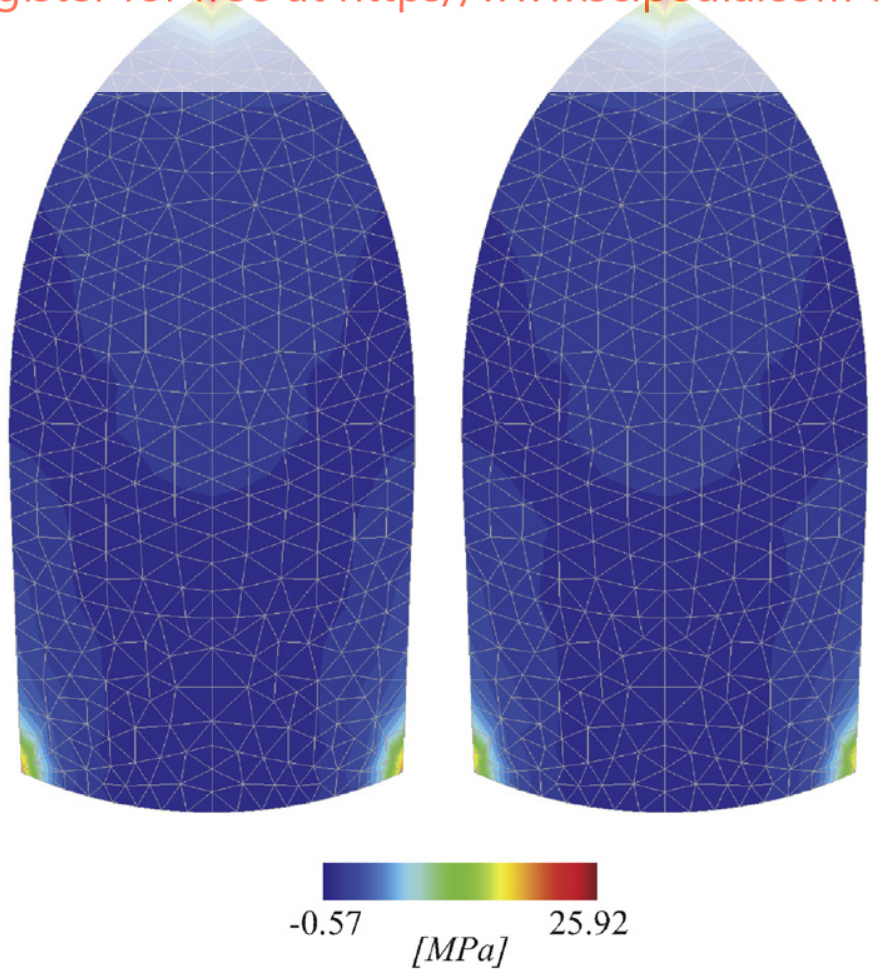

Fig. 20. Second Piola-Kirchhoff stresses. Left: contours of $S_{x x}$. Right: contours of the maximum axial stress $S_{1}$.

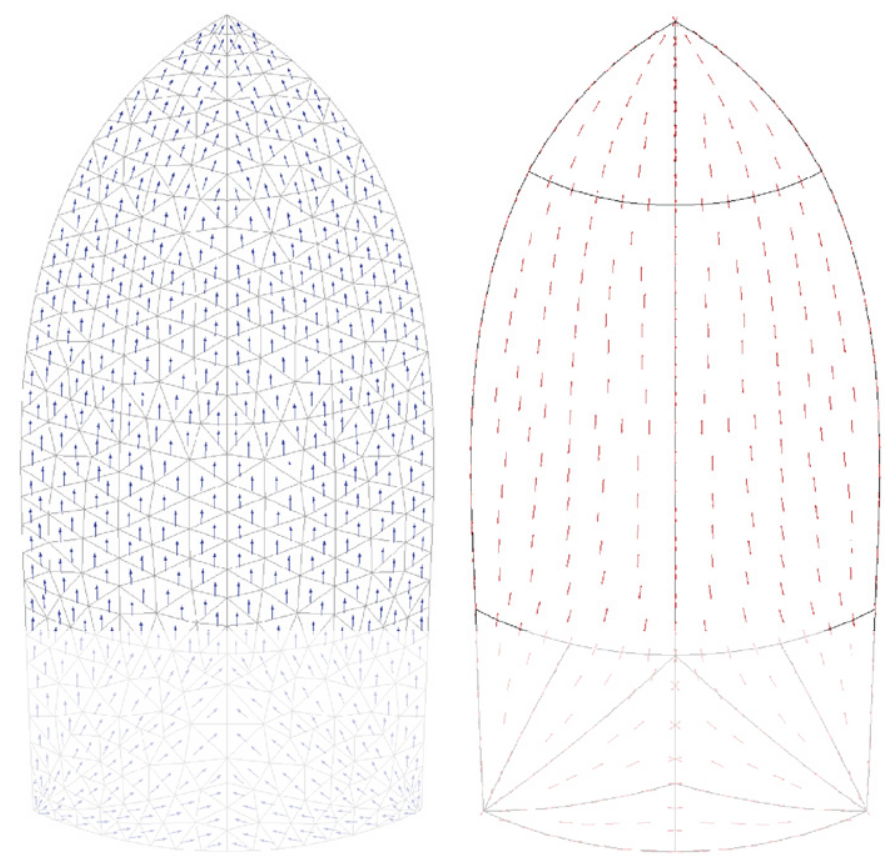

Fig. 21. Optimal fiber orientation vectors (left) and $1^{\text {st }}$ principal stress directions (right). Note the coincidence of both vector fields.

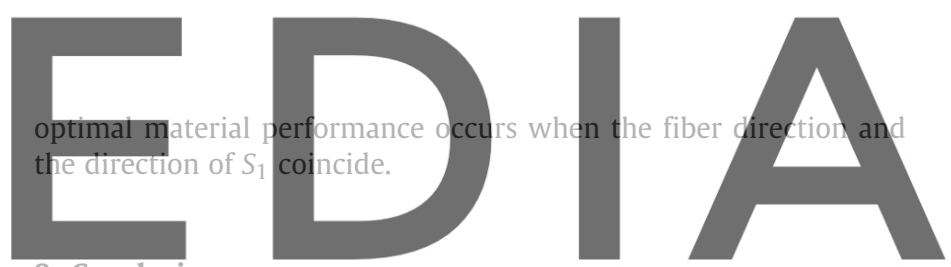

8. Conclusions

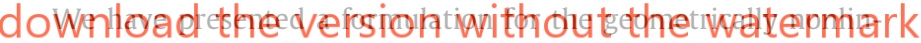
ear analysis of orthotropic membrane structures. The formulation is based on a continuum mechanics approach leading to an expression of the vintual internal vroik in curvilinear coordinates. A nevv triangular finite element formulation based on the principal fiber orientation has been presented.

The idea to use the fiber orientation of the material for our analysis comes from the need to define a direction for the principal fiber of the material in order to manufacture the structure. The fiber orientation approach has many advantages, which can be stated as follows:

- It is based on an idea which is very simple to understand.

- Allows adequate postprocessing of the problem since any number of patches that conform the structure can be postprocessed with different local orientations.

- It allows to perform analysis with orthotropic and composite materials including membrane structures having an initially out-ofplane configuration.

- Prestressed forces can be easily accounted for in the membrane structure.

The inclusion of the fiber orientation allows to improve the standard membrane formulation which can be considered as a particular case of the more general orthotropic formulation. An algorithm for modeling the wrinkling behavior of membrane structures has also been described.

The examples presented show the potential of the new membrane formulation for analysis of orthotropic and prestressed membrane structures. 


\section{References}

[1] O.C. Zienkiewicz, R.L. Taylor, The Finite Element Method, fourth ed., vols. 1 and 2, McGraw-Hill, London, 1989.

[2] R.D. Cook, D.S. Malkus, M.E. Plesha, Concepts and Applications of Finite Element Analysis, third ed., Wiley, New York, 1989.

[3] E. Oñate, Structural Analysis by the Finite Element Method. Vol. 2: Beams, Plates and Shells, CIMNE-Springer, 2008.

[4] J.C. Simo, D.D. Fox, On a stress resultant geometrically exact shell model. Part i: formulation and optimal parametrization, Comput. Methods Appl. Mech. Eng. 72 (1989) 267-304.

[5] J.C. Simo, D.D. Fox, M.S. Rifai, On a stress resultant geometrically exact shell model. Part iii: computational aspects of the nonlinear theory, Comput. Methods Appl. Mech. Eng. 79 (1990) 21-70.

[6] N. Bütchter, E. Ramm, D. Roehl, Three-dimensional extension of non-linear shell formulations based on the enhanced assumed strain concept, Int. J. Numer. Methods Eng. 37 (1992) 2551-2568.

[7] M. Braun, M. Bischoff, E. Ramm, Nonlinear shell formulations for complete three-dimensional constitutive laws include composites and laminates, Comput. Mech. 15 (1994) 1-18.

[8] J. Bonet, R.D. Wood, J. Mahaney, P. Heywood, Finite element analysis of air supported membrane structures, Comput. Methods Appl. Mech. Eng. 190 (2000) 579-595.

[9] K. Lu, M. Accorsi, J. Leonard, Finite element analysis of membrane wrinkling, Int. J. Numer. Methods Eng. 50 (2001) 1017-1038.

[10] R.L. Taylor, Finite element analysis of membrane structures, Internal CIMNE report, Barcelona, 2001.

[11] J.G. Valdés, Analysis of membrane structures. Technical Report IT 386, CIMNE, Barcelona, 2002 (in Spanish).

[12] R. Rossi, Light-weight structures. Numerical analysis and coupling issues, Ph.D. Thesis, University of Bologna, Italy, 2005.

[13] R. Levy, W. Spillers, Analysis of Geometrically Nonlinear Structures, Chapman \& Hall, London, 1995.

[14] T.A. Raible, Concepts for nonlinear orthotropic material modeling with applications to membrane structures, Ph.D. Thesis, Universität Hannover, Institut für Baumechanik und Numerische Mechanik, 2003.

[15] A.J. Gil, Finite element analysis of prestressed hyperelastic saint venantkirchhoff membranes under large deformations, in: E. Oñate, B. Kröpling
(Eds.), Textile Composites and Inflatable Structures, Barcelona, CIMNE, 2003, pp. 206-211.

[16] R. Wüchner, K.-U. Bletzinger, Stress-adapted numerical form finding of prestressed surfaces by the updated reference strategy, Int. J. Numer. Methods Eng. 64 (2005) 143-166.

[17] J.G. Valdés, E. Oñate, J.M. Canet, Assignment of principal fibers direction to analyze orthotropic membranes, in: J. Mora, E. Escolano, M. Pasenau, R. Ribó, J.S. Pérez (Eds.), Conference on Advances and Applications of GiD, Barcelona, CIMNE, 2004, pp. 105-108.

[18] J.G. Valdés, E. Oñate, Orthotropic rotation-free basic thin shell triangle, Comput. Mech. (2009), doi:10.1007/s00466-009-0370-y.

[19] J.G. Valdés, Nonlinear analysis of orthotropic membrane and shell structures including fluid-structure interaction, Ph.D. Thesis, Technical University of Catalunya 〈http://www.tdcat.cesca.es/TDX-1126107-193535〉, 2007.

[20] M. Farrashkhalvat, J.P. Miles, Basic Structures Grid Generation, ButterworthHeinemann, Oxford, 2003.

[21] M.A. Crisfield, Non-linear Finite Element Analysis of Solids and Structures, vols. 1 and 2, Wiley, Chichester, 1991.

[22] C. Decolon, Analysis of Composite Structures, Hermes Science Publications, Paris, 2000.

[23] A. Tessler, D.W. Sleight, J.T. Wang, Effective modeling strategies for nonlinear shell analysis of thin membranes exhibiting structural wrinkling, J. Spacecraft Rockets, AIAA 42 (2) (2005) 287-298.

[24] D.G. Roddeman, J. Drukker, C.W.J. Oomens, J.D. Janssen, The wrinkling of thin membranes: Part i-theory, ASME J. Appl. Mech. 54 (1987) 884-887.

[25] D.G. Roddeman, J. Drukker, C.W.J. Oomens, J.D. Janssen, The wrinkling of thin membranes: Part ii-numerical analysis, ASME J. Appl. Mech. 54 (1987) $888-892$.

[26] A. Jarasjarungkiat, R. Wüchner, K.-U. Bletzinger, A wrinkling model based on material modification for isotropic and orthotropic membranes, Comput. Methods Appl. Mech. Eng. 197 (6) (2008) 773-788.

[27] J. Chung, G.M. Hulbert, A time integration algorithm for structural dynamics with improved numerical dissipation: the generalized- $\alpha$ method, J. Appl. Mech. 60 (1993) 371-375.

[28] T.E. Tezduyar, S. Sathe, K. Stein, Solution techniques for the fully discretized equations in computation of fluid-structure interactions with space-time formulations, Comput. Methods Appl. Mech. Eng. 195 (2006) 5743-5753. 